\title{
Blazhko effect in Cepheids and RR Lyrae stars
}

\author{
Róbert Szabó \\ Konkoly Observatory, Research Center for Astronomy and Earth Sciences \\ of the Hungarian Academy of Sciences \\ Konkoly Thege Miklós út 15-17. \\ H-1121, Budapest, Hungary \\ email: szabo.robert@csfk.mta.hu
}

\begin{abstract}
The Blazhko effect is the conspicuous amplitude and phase modulation of the pulsation of RR Lyrae stars that was discovered in the early 20th century. The field of study of this mysterious modulation has recently been invigorated thanks to the space photometric missions providing long, uninterrupted, ultra-precise time-series data. In this paper I give a brief overview of the new observational findings related to the Blazhko effect, such as extreme modulations, irregular modulation cycles and additional periodicities. I argue that these findings together with dedicated ground-based efforts provide us now with a fairly complete picture and a good starting point to theoretical investigations. Indeed, new, unpredicted dynamical phenomena have been discovered in Blazhko RR Lyrae stars, such as period doubling, high-order resonances, threemode pulsation and low-dimensional chaos. These led to the proposal of a new explanation to this century-old enigma, namely a high-order resonance between radial modes. Along these lines I present the latest efforts and advances from the theoretical point of view. Lastly, amplitude variations in Cepheids are discussed.
\end{abstract}

Keywords. stars: variables: RR Lyrae, stars: variables: Cepheids, Blazhko effect, stars: individual: RR Lyr, V445 Lyr, V473 Lyr, $\alpha$ UMi, pulsation, hydrodynamics, Kepler

\section{Introduction}

Although representatives of several pulsating variable types show amplitude modulation, the Blazhko effect of RR Lyrae stars is unique in many respects. First, the percentage of modulated fundamental mode RR Lyrae stars can be as high as 50\% (Konkoly Blazhko Survey, Jurcsik et al. 2009). The modulation time scale ranges from a few days to several years. Second, besides amplitude modulation, simultaneous phase modulation (or, equivalently period variation) is always present in modulated RRab stars (Benkö et al. 2010). The third point is a freshly distilled lesson from a series of recent discoveries: the modulation of the high-amplitude nonlinear pulsational modes are accompanied by several dynamical phenomena (period doubling, resonances, chaos) and this feature makes the Blazhko effect in RR Lyrae stars a unique feature among the classes of pulsating variables. We note in passing that these dynamical effects have a special role in RR Lyrae stars, even the Blazhko effect itself might have a dynamical origin (i.e. resonance between radial modes, see later).

The Blazhko effect was discovered by S. Blažko more than a century ago (Blažko 1907) when he could not fit the period of RW Dra with a constant value (phase modulation). A few years later H. Shapley (Shapley 1916) noticed the different heights of observed maxima of RR Lyrae, the prototype, discovering the amplitude modulation.

Only a few years have passed since the latest major review on the topic (Kovács 2009). By coincidence, the launch of Kepler (Borucki et al. 2010) preceded this excellent review 
only by a few months. This space telescope has opened a completely new window to the intricate behavior of the Blazhko-modulated RR Lyrae stars. Therefore I focus on the enormous progress that has been made since Kovács' (2009) review.

A very important aspect is that without assuming any special physical mechanism, the mathematical description of the modulation of a carrier wave (the pulsation in our case) can be elegantly derived from simple considerations (Benkő et al. 2011, Szeidl \& Jurcsik 2009). This kind of description admittedly does not convey any knowledge about the underlying physical mechanism, but helps to discriminate between physical interpretations. In particular, instead of detecting only one side frequency (doublet) or symmetric sidepeaks (triplet) (Alcock et al. 2003) or sometimes quintuplets (Hurta et al. 2008) around the dominant frequency and its harmonics from ground-based observations, with space data we can find a large (in theory infinite) number of side peaks in Blazhko RR Lyrae stars. For example, in the Fourier-spectrum of V1127 Aql up to 6-8 side peaks on both sides could be detected around the dominant frequency and its harmonics with CoRoT (Chadid et al. 2010). The side peak structure is only one aspect of the modulation. Many more unexpected results came from space photometric missions, and I continue with highlighting some of the spectacular Kepler observational results.

\section{The Kepler revolution - observational results}

The homogeneous photometric data for more than 200000 stars delivered by Kepler is unprecedented and proved to be a treasure trove both for transiting exoplanet finding and stellar astrophysics. The ultra-high precision (e.g. $8 \mathrm{ppm}$ per point precision for the bright, heavily saturated RR Lyr, the prototype, Kolenberg et al. 2011) exceeds by orders of magnitude what has been possible from the ground. In addition, the 4-yearlong, quasi-uninterrupted monitoring allowed the exploration of a completely new region of parameter space. For many discoveries not the precision, but the continuity was the most important factor. The continuous observations were interrupted only for a few hours per month for data downloading or a few days in case of shorter and up to two weeks for longer technical problems. Some stars that fell on the dead Module 3 show regular, 3-month-long gaps annually.

With this extraordinary instrumental setup apart from a few RRc stars (Moskalik et al., in preparation) forty-four RRab stars were observed, 17 of them being Blazhkomodulated, which is close to $40 \%$. A few more RR Lyrae stars have been discovered either serendipitously (background contamination) or by meticulous investigations by dedicated citizen scientists (PlanetHunters) and will be added to the list soon. Unfortunately, after four years of operations Kepler has lost its second reaction wheel, which degrades the pointing stability, hence photometric precision, as well.

\subsection{Extreme modulations - modulation extremes}

One of the most intriguing features that Kepler was able to show us is the huge variety of modulation shapes, and in some cases its cycle-to-cycle variations. There are extreme cases with large variability in the modulation cycles, while in other stars the modulation seems to be more or less regular. The poster child of the rapid variations in modulation characteristics is V445 Lyr (Guggenberger et al. 2012), where significant variations are observed from cycle to cycle. In addition, drastic light curve shape variation is evident: in Blazhko-maxima the light curve is that of a normal RRab star, but in Blazhko-minima the amplitude is an order of magnitude less, and it transforms into a double-maxima, irregular light curve, that does not resemble any RRab light variations. A sibling of this object is CoRoT 105288363 (Guggenberger et al. 2011). 
Another important revelation was the multiperiodic nature of the modulations. One of the best-observed examples found by ground-based observations is CZ Lac (Sódor et al. 2011), which was followed by many more examples from Kepler. In fact, based on the four-year long Kepler data, it seems that most of the modulated RRab stars show multiple periodicities in their modulations, except the longest-period ones, where more data would be necessary to establish any multi-periodicity (Benkő et al., in preparation). In CZ Lac two simultaneous modulation periods were detected and both changed its period from one observing season to another. This fact, along with the multiperiodic/irregular behavior of the modulation, immediately raises the question whether the underlying dynamics is chaotic, and the changing, seemingly multiperiodic modulations are simply the manifestations of a chaotic behavior. There are indications that this scenario might indeed be plausible (see below).

\subsection{The unexpected beauty: period doubling}

One of the most remarkable discoveries on the Blazhko-front has been the detection of a well-known dynamical phenomenon, the period doubling (hereafter PD), which has never been observed in RR Lyrae stars before Kepler. PD was easily noticed in the first long-cadence (29.5-min) Kepler light curves (Kolenberg et al. 2010, Szabó et al. 2010) as alternating maxima in some of the Blazhko RR Lyrae stars. PD (or period-2 state) manifests itself in the frequency domain as the presence of half-integer frequencies between the dominant pulsational frequency and its harmonics. After the first detection, it turned out that the majority of Blazhko-modulated stars shows PD, at least temporarily. In each case, the strength of $\mathrm{PD}$ varies with time, and it can vanish for long time intervals, which partly explains why it could remain unnoticed before the space photometry era. Anyhow, the fact that PD is present in most of the Blazhko RRab stars, and has never been seen in non-modulated RR Lyrae stars, even with the precision allowed by Kepler (Szabó et al. 2010, Nemec et al. 2011), demonstrates boldly that there should be a strong connection between PD and the Blazhko effect itself. In Fig. 1 we see a characteristic and very strong PD phase of RR Lyrae, the eponym of its class.

\subsection{Additional periodicities in modulated RR Lyrae stars}

Interestingly, in addition to the traditional frequency solution of the Blazhko RR Lyrae stars (dominant mode, its harmonics and modulation side peaks), extra frequencies can be frequently found in Blazhko RR Lyrae stars if space photometric data of sufficiently high precision are available. These frequencies are present in non-Blazhko stars as well (Moskalik 2013 and Moskalik, these proceedings) and their origin (if pulsation) can be either radial (Benkő et al., these proceedings) or non-radial modes. However, in Blazhko RRab stars most of these frequencies tend to show up around the radial low-order overtones, namely around the predicted frequencies of the first (O1) and second (O2) overtones (see e.g. Benkö et al. 2010). As has been shown by Wojtek Dziembowski, nonradial modes can be unstable in RR Lyrae star models (Dziembowski 1977). Such modes are most easily excited in the vicinity of radial overtones (Van Hoolst et al. 1998), so the radial/nonradial dilemma cannot be readily solved. It is worth noting in this context that in at least one case (RR Lyrae itself) we were able to prove that the radial O1 mode is excited (see Sect. 3.2). 


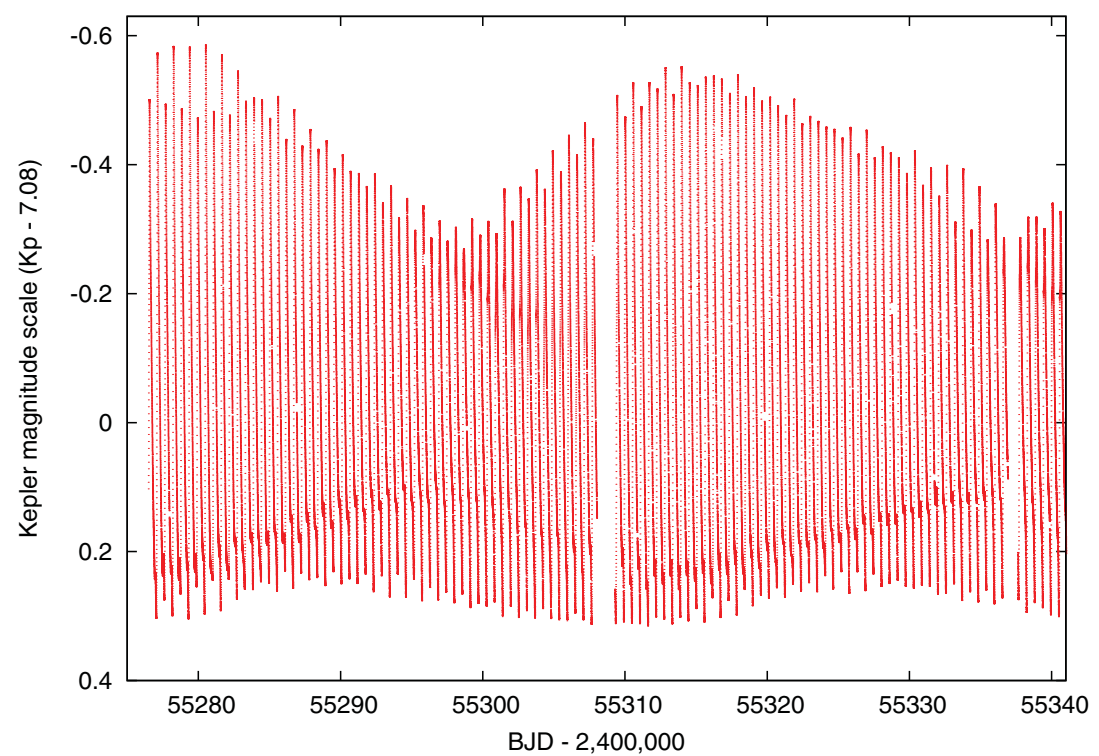

Figure 1. Q5 short-cadence (1-min sampling) Kepler flux data of RR Lyrae, the prototype converted to the Kepler magnitude scale and shifted by an arbitrary number. Period doubling is persistent throughout this 2-month section. Note the large difference between the heights of consecutive maxima at the beginning. The plot contains more than 91000 individual data points.

\section{The Kepler revolution - theoretical aspects}

How can these recent findings fit into a coherent theoretical framework? Will eventually all the pieces of the Blazhko jigsaw puzzle fall into place? In what follows I am presenting the latest theoretical advancements inspired by the discoveries discussed above.

\subsection{Resonances, resonances, resonances}

Resonances play a crucial role in Cepheids, but because of the narrower region in the parameter space occupied by RR Lyrae stars, resonances were thought to play a negligible role in these high-amplitude horizontal branch pulsators. Surprisingly, Szabó et al. (2010) and Kolláth et al. (2011) were able to prove by computing hydrodynamic models that the origin of the PD is undoubtedly a high-order (9:2) resonance between the fundamental mode and a high-order radial (strange) overtone. This resonance is at work for a relatively large portion of the mass - luminosity - effective temperature - metallicity parameter space. Although the 8 th and the 10 th radial overtones can also be trapped in the outer regions, hence not so heavily damped or even excited, a large survey of models demonstrated unambiguously that the ninth overtone is coupled very strongly to the fundamental mode and causes the period doubling. A series of period-doubling bifurcations can lead to chaos. We were able to find such bifurcation cascades in RR Lyrae models. Further full hydro computations showed that our models often approach other high-order resonant states, like 14:19, 20:27, etc., between the fundamental and the first overtone modes, creating a huge variety of complex dynamical behavior.

\subsection{Triple-mode states}

One such dynamical state is a three-mode condition. Molnár et al. (2012) investigated the hydro models with PD that showed period-6 behavior as well (where the height of maxima repeats after 6 pulsation periods), indicating that the model can temporarily 
be close to the $3: 4$ resonance between the fundamental and first overtone. This period- 6 characteristic was found in the Kepler observations of RR Lyr, the prototype. Based on the models, the detection of the first overtone was predicted with low amplitude, and indeed Q5-Q6 Kepler data of RR Lyrae showed the O1 frequency with high significance (Molnár et al. 2012). Based on this result, the presence of the first radial overtone itself is well established, but additional, non-radial modes cannot be completely ruled out either. This three-mode state (fundamental, O1 and O9) on the one hand represents a new pulsational behavior dissimilar to the well-known double-mode (RRd) pulsators, and on the other hand enables an even more diverse bonanza of complex dynamical states, including chaos (Plachy et al. 2013).

\subsection{Radial resonance as an explanation of the Blazhko effect}

Buchler \& Kolláth (2011) moved forward and showed using the amplitude equation formalism that if the 9:2 resonance between the fundamental mode and the 9th overtone is present, then in a large part of parameter space regular, irregular, and even chaotic modulations occur naturally. This result is a very important step towards the understanding of the Blazhko effect. The final step of proving the concept with full hydrodynamics models still remains to be done, but it is worth mentioning here that Smolec \& Moskalik (2012) were able to produce modulated hydrodynamic models of BL Her stars in the presence of period doubling. The radial resonance paradigm is currently the most tenable, and the only one that is backed up by full hydrodynamic models. Additional resonances and the presence of nonradial modes are also highly probable, and finding them should be of prime priority. Much work is required to fully validate the resonance model, e.g. to reconcile the models with occurrence rates and observed quantities, and to work out a similar mechanism for Blazhko RRc stars.

\subsection{Low-dimensional chaos in the Blazhko modulation}

Based on recent work of Plachy et al. (in preparation), there are indications that the modulation of some of the Kepler RRab stars with shorter modulation periods may be the result of low-dimensional chaos. The work is based on advanced mathematical methods, like global flow reconstruction and compares various return maps of the observed data to synthetic, chaos-generated data sets. The key factor for the successful application of the method is the observed number of modulation cycles. RR Lyrae stars having the shortest period (20-30 days) modulations accumulated enough cycles during Kepler's 4 -year operation to be suitable for such analyses. For the long-period Blazhko stars more observational data would be essential. If Kepler's operation is extended and it continues to observe the same field in two-wheel mode (e.g. to detect transit-timing variation of the already discovered planetary systems), then there is a good chance that a few Kepler Blazhko stars will be monitored as well (Molnár et al. 2013a). We emphasize here again that the radial resonance model (Buchler \& Kolláth 2011) is able to predict chaotic modulation cycles, hence observations seem to confirm the theoretical predictions.

\section{Amplitude variations in Cepheids}

Although several flavors of amplitude variation have been detected in Cepheids, these do not always resemble the Blazhko effect seen in RR Lyrae stars. One such example is the large number $(19 \%)$ of first overtone - second overtone (O1/O2) double-mode Cepheids in the Large Magellanic Cloud that show long-period $\left(P_{\bmod }>700 \mathrm{~d}\right)$, anti-correlated amplitude modulation discovered by Moskalik \& Kołaczkowski (2009). The true occurrence rate can be higher, considering the finite length of the OGLE-II observations and 

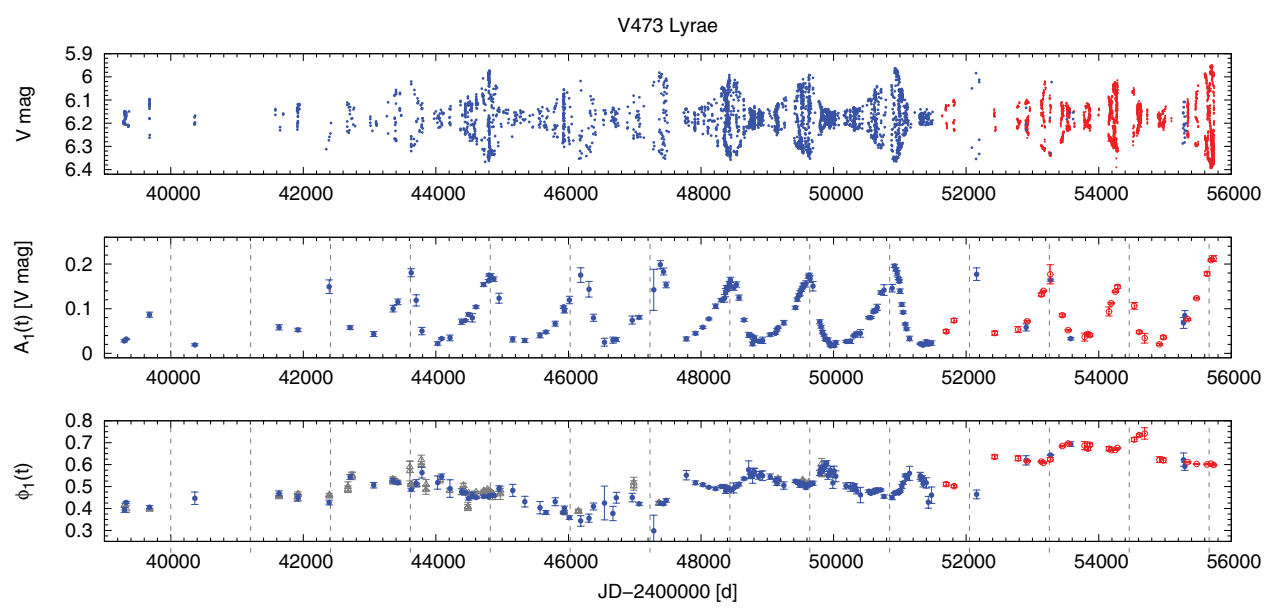

Figure 2. Blazhko-like variations of V473 Lyr. Upper panel: light curve, middle panel: amplitude variation $\left(A_{1}\right.$ term of the Fourier-decomposition), bottom panel: phase variation. The vertical dashed lines denote the 1205-day modulation cycles. The full circles are $V$ data, open symbols are scaled Strömgren $v$ observations to match the amplitudes in $V$. In the bottom plot the triangles are $\mathrm{O}-\mathrm{C}$ data taken from the literature (Molnár et al. 2013b and references therein.)

a significant number of $\mathrm{O} 1 / \mathrm{O} 2$ double-mode Cepheids showing amplitude variations on even longer time scales. The origin of this phenomenon is currently unknown.

Another prominent example is the amplitude change seen in the famous Cepheid $\alpha \mathrm{UMi}$ or Polaris (Turner et al. 2005). The light as well as the radial velocity amplitude of this overtone Cepheid had been decreasing until the mid-nineties, then it rebounded and it has been increasing since then (Bruntt et al. 2008, Spreckley \& Stevens 2008). It is not clear whether the variation is secular or cyclic. Obviously, more observations are required to answer this question. The cause of the variation is also a matter of debate, the suggested mechanisms range from variations in the stellar structure due to evolution through the presence of an additional (presumably) nonradial mode. Unless it is an extremely longperiod modulation, there is no indication that the amplitude variation of Polaris would be related to the Blazhko effect in RR Lyrae stars.

Contrary to Polaris, V473 Lyrae (Burki et al. 1986) is a possible example for a Blazhko Cepheid. The characteristics of the light variation of this presumably second overtone Cepheid are almost identical to the Blazhko-modulated RRab stars (Molnár et al., in preparation). The argument is based on a recent analysis of new photometric data (Molnár et al. 2013b) showing Blazhko-like amplitude modulation and simultaneous period variation, a distinctive characteristic of Blazhko RR Lyrae stars (see Fig. 2).

As a side note we add that the variations we discussed in this section fit the trend that was found on shorter time scales (Evans et al., these proceedings and references therein), namely a more pronounced tendency for instability in the oscillations of overtone pulsators compared to fundamental-mode Cepheids.

\section{The future of the Blazhko research field}

From the observational side the success story of space photometry is expected to be continued. NASA's TESS (Transiting Exoplanet Survey Satellite, Ricker et al. 2010) mission with a launch scheduled for 2017 will monitor the whole sky (it will observe a given field for 27 days) searching for short-period planets around bright stars. The design of four large field-of-view cameras will allow good photometric precision. A small 
fraction of the sky around the ecliptic poles will be observed continuously, which will be an excellent hunting ground for longer period Blazhko stars. The European counterpart of TESS is the PLATO (PLAnetary Transits and Oscillations of Stars) mission (Rauer et al. 2013). If selected, the arrangement of 32 normal and 2 fast telescopes on a common platform and the judiciously designed overlap in the monitored fields between the telescopes will provide large field-of-views and high precision. During the nominal 5-yr mission half of the sky could be covered with long (from months to up to a year) staring phases peppered with shorter step-and-stare intervals. The earliest launch date for PLATO is 2024. In both cases the missions will most probably avoid the overcrowded low galactic latitude regions. Needless to say, both space photometric projects can continue the pioneering work of CoRoT and Kepler and contribute to the understanding of the long-standing Blazhko-problem.

There is great potential in spectroscopic observations as well. Systematic, dedicated studies covering several Blazhko cycles are rare. Promising results have already been published recently, e.g. the detection of He I and He II lines in Blazhko stars (Preston 2009, 2011, Gillet et al. 2013, see also the contributions of Guggenberger and Kolenberg, these proceedings). Understanding the complicated dynamics of the pulsating atmosphere and the study of any non-spherical asymmetries (presence of nonradial modes for instance) would greatly benefit from such investigations.

On the theoretical front, one-dimensional hydrocodes, e.g., the Budapest-Florida code (Kolláth et al. 2002), or the Warsaw code (Smolec \& Moskalik 2008) still have a role in understanding physical concepts and mechanisms, especially in the light of the radial resonance paradigm. It has become increasingly clear that multi-dimensional codes (Kupka, these proceedings, Geroux \& Deupree 2011, 2013, and these proceedings) will greatly advance our understanding of stellar pulsation in general and of the Blazhko effect in particular in the near future. When long simulations with many different initial conditions become feasible in two or three-dimensions, the self-consistent treatment of convection, the ability of modeling nonradial motions (or even modes) will make these hydrocodes excellent tools in helping to convey a better picture of the mode selection mechanism, the interaction between pulsation and convection, and the modulation mechanism itself. Clearly, the modeling of the these inherently three-dimensional objects and dynamical processes in multi-dimensions requires an enormous amount of work, but I strongly believe that a better understanding of RR Lyrae stars and the Blazhko effect is well worth the effort.

\section{Acknowledgements}

The author gratefully acknowledges the Lendület-2009 Young Researchers' Program and the János Bolyai Research Scholarship of the Hungarian Academy of Sciences, the HUMAN MB08C 81013 grant of the MAG Zrt., the Hungarian OTKA grant K83790, the KTIA URKUT_10-1-2011-0019 grant, the European Community's FP7/2007-2013 programme under grant agreement no. 269194 (IRSES/ASK), and the IAU for the travel grant. The inspiring discussions and influential work of Wojtek Dziembowski in the field of stellar pulsation are also thankfully acknowledged. Fruitful discussions with Z. Kolláth, L. Molnár, E. Plachy, J. M. Benkő, K. Kolenberg, P. Moskalik, and R. Smolec are appreciated. The author wishes to dedicate this review to the memory of J. Robert Buchler.

\section{References}

Alcock, C., Alves, D. R., Becker, A., et al. 2003, ApJ, 598, 597

Benkő, J. M., Kolenberg, K., Szabó, R., et al. 2010, MNRAS, 409, 1585 
Benkő, J. M., Szabó R. \& Paparó, M. 2011, MNRAS, 417, 974

Borucki, W. J. Koch, D., Basri, G., et al. 2010, Science, 327, 977

Bruntt, H., Evans, N. R., Stello, D., et al. 2008, ApJ, 683, 433

Blažko, S. 1907, AN, 175, 327

Buchler, J. R. \& Kolláth, Z. 2011, ApJ, 731, 24

Burki, G., Schmidt, E. G., Arellano Ferro, A., et al. 1986, A\&A A, 168, 139

Chadid, M., Benkő, J. M., Szabó, R., et al. 2010, A\&AA, 510, A39

Dziembowski, W. 1977, AcA 27, 95

Geroux, C. M. \& Deupree, R. G. 2011, ApJ, 731, 18

Geroux, C. M. \& Deupree, R. G. 2013, ApJ, 771, 113

Gillet, D., Fabas, N., \& Lèbre, A. 2013, A\& 4 A 553, A59

Guggenberger, E., Kolenberg, K., Poretti, E., et al. 2011, MNRAS, 415, 1577

Guggenberger, E., Kolenberg, K., Nemec, J. M., et al. 2012, MNRAS, 424, 649

Hurta, Zs., Jurcsik, J., Szeidl, B., \& Sódor, Á. 2008, AJ, 135, 957

Jurcsik, J., Sódor, Á., Szeidl, B., et al. 2009, MNRAS, 400, 1006

Kolenberg, K., Szabó, R., Kurtz, D. W., et al. 2010, ApJ, 713, L198

Kolenberg, K., Bryson, S., Szabó, R., et al. 2011, MNRAS, 411, 878

Kolláth, Z., Buchler, J. R., Szabó, R., \& Csubry, Z. 2002, A\&̛A, 385, 932

Kolláth, Z., Molnár, L., \& Szabó, R. 2011, MNRAS, 414, 1111

Kovács, G. 2009, AIP-CP, 1170, 261

Molnár, L., Kolláth, Z., Szabó, R., et al. 2012, ApJ, 757, L13

Molnár, L., Szabó, R., Kolenberg, K., et al. 2013a, arXiv: 1309.0740

Molnár, L., Szabados, L., Dukes, R. J. Jr, Győrffy, Á., \& Szabó, R. 2013b, AN, in press (arXiv: 1309.2108)

Moskalik, P. 2013, in: J. C. Suárez, R. Garrido, L. A. Balona, \& J. Christensen-Dalsgaard (eds.), Stellar Pulsations, Astrophysics and Space Science Proceedings, Vol. 31 (Berlin, Heidelberg: Springer-Verlag), p. 103

Moskalik, P. \& Kołaczkowski, Z. 2009, MNRAS, 394, 1649

Nemec, J. M., Smolec, R., Benkő, J. M., et al. 2011, MNRAS, 417, 1022

Plachy, E., Kolláth, Z., \& Molnár, L. 2013, MNRAS, 433, 3590

Preston, G. W. 2009, A\&A, 507, 1621

Preston, G. W. 2011, AJ, 141, 6

Rauer, H., Catala, C., Aerts, C., et al. 2013, Experimental Astronomy, submitted (arXiv: 1310.0696)

Ricker, G. R., Latham, D. W., Vanderspek, R. K., et al. 2010, BAAS, 42, 459

Shapley, H. 1916, ApJ, 43, 217

Smolec, R. \& Moskalik, P. 2008, AcA, 58, 193

Smolec, R. \& Moskalik, P. 2012, MNRAS, 426, 108

Spreckley, S. A. \& Stevens, I. R. 2008, MNRAS, 388, 1239

Sódor, Á., Jurcsik, J., Szeidl, B., et al. 2011, MNRAS, 411, 1585

Szabó, R., Kolláth, Z., Molnár, L., et al. 2010, MNRAS, 409, 1244

Szeidl, B. \& Jurcsik, J. 2009, CoAst, 160, 17

Turner, D. G., Savoy, J., Derrah, J., Abdel-Sabour, A.-L. M., \& Berdnikov, L. N. 2005, PASP, 117,207

Van Hoolst, T., Dziembowski, W. A., \& Kawaler, S. D. 1998, MNRAS, 297, 536 